\title{
UMA ESTRATÉGIA PARA O MONITORAMENTO DA ANCHOITA (ENGRAULIS ANCHOITA, ENGRAULIDIDAE, PISCES): O ÚLTIMO ESTOQUE DE PEQUENO PEIXE PELÁGICO CAPAZ DE SUSTENTAR UMA PESCA COMERCIAL DE MÉDIA ESCALA EM ÁGUAS BRASILEIRAS
}

\author{
CAROLINE VIEIRA COOKE, LAURO SAINT PASTOUS MADUREIRA \\ Universidade Federal do Rio Grande - Laboratório de Tecnologia Pesqueira e Hidroacústica, Rio Grande - RS - Brasil. CEP: $96203-000$ - \\ Caixa postal: 474 carucooke@gmail.com; doclsm@furg.br
}

\begin{abstract}
RESUMO
O estoque bonaerense de Engraulis anchoita distribui-se entre as costas da Argentina, Uruguai e do sul e sudeste do Brasil. Atualmente, vêm chamando a atenção de empresas de pesca brasileiras, pela elevada abundância e nível de explotação muito abaixo de seu potencial. A anchoita é atualmente a única espécie na costa brasileira com potencial significativo de aproveitamento comercial em pescarias de média escala (acima de 30 mil toneladas por ano). Somente os países do Prata pescam este estoque e há grande probabilidade de um aumento da pressão pesqueira sobre esta espécie nos próximos anos, tornando-se um recurso comercial também no Brasil, desde que solucionados os problemas de conservação a bordo e mercado. Devido à importância da anchoita como espécie chave no ecossistema do Atlântico Sul Ocidental, e considerando os recentes colapsos da pesca de estoques de pequenos pelágicos cogenéricos em todo o planeta, há necessidade da elaboração de um plano de manejo, a ser implantado pelo Brasil, o que levaria a um raro caso de um recurso manejado desde o início de sua explotação. Neste trabalho, é descrito o desenho amostral presumidamente mais adequado para uma série de cruzeiros de estimativas acústicas de abundância da anchoita e sugere-se que esta estratégia venha a ser usada para subsidiar futuros planos de manejo desta espécie, que garantam sua sustentabilidade a longo prazo.
\end{abstract}

PALAVRAS CHAVE: desenho de cruzeiro, Engraulis anchoita, estoque bonaerense, plano de manejo, metodologia hidroacústica.

\begin{abstract}
Strategy action to monitor Anchoita (Engraulis anchoita, Engraulididae, Pisces): the last small pelagic fish stock able to support a medium size scale fishery in Brazilian waters.

The bonaerence stock of Engraulis anchoita is distributed along the Argentinean and Uruguayan coasts as well as on the south and southeast of Brazilian coast. In Brazil it is attracting the attention of the fishing industry due to its great abundance and exploitation quite below its potential. Nowadays, there are no other species in Brazil with significant potential to sustain a commercial middle scale fishery (above 30 thousand tons/year). Only Argentina and Uruguay are fishing this stock. However, there is a great probability of an increasing pressure to fish this species by the Brazilian fleet, since on board conservation and market problems are solved. Due to the importance of anchoita as a key species in the ecosystem of the Southern Atlantic Ocean and considering the recent collapses in the stock of cogeneric small pelagics all over the world, it becomes necessary to introduce a management plan in Brazil. This would lead to one of the few cases in which exploitation of fishery resources is being managed from the very beginning. In this work, the presumed most adequate strategic survey design is described to estimate anchoita abundance with acoustics, and it is also suggested its use to subsidize future management plans.
\end{abstract}

KEYWORDS: survey design, Engraulis anchoita, bonaerense stock, management plan, hydroacoustic methodology.

\section{INTRODUÇÃO}

Uma espécie de pequeno peixe pelágico vem chamando a atenção de pesquisadores e empresas de pesca nos últimos anos, devido a sua grande abundância em determinadas épocas do ano nas regiões sudeste e sul da costa brasileira, e nível de explotação muito abaixo de seu potencial, além de sua importância como forrageira (Castello \& Castello, 2003). A espécie em questão, conhecida popularmente como anchoita (Engraulis anchoita, Engraulididae, Pisces) (Hubbs \& Marini, 1935), ocorre principalmente sobre a plataforma continental, desde águas mais rasas que $10 \mathrm{~m}$ até aproximadamente $200 \mathrm{~m}$ (Bakun \& Parrish, 1991) e tolera um amplo espectro de temperatura $\left(8 \mathrm{a} 23^{\circ} \mathrm{C}\right)$ e salinidade $(14 \mathrm{a}$ 35). Evidências baseadas em parâmetros biológicos (Castello, 2005) sugerem a ocorrência de três subpopulações: 1) Patagônica, entre $47^{\circ}$ e $41^{\circ} S$; 2) Bonaerense, entre $41^{\circ} \mathrm{S}$ e $29^{\circ} \mathrm{S}$, e 3) uma população ocorrendo no sudeste brasileiro, entre $29^{\circ} \mathrm{S}$ e $22^{\circ} \mathrm{S}$.

A sub-população Bonaerense, compartilhada entre Argentina, Uruguai e Brasil, representa a de maior abundância (Hansen \& Madirolas, 1996) e, segundo Madureira et al. (2005), o limite oceânico de distribuição em águas do sul do Brasil parece estar relacionado à Frente Subtropical de Plataforma que divide águas frias de baixa salinidade (Água SubAntártica de Plataforma) de águas quentes e de alta salinidade (Água Subtropical de Plataforma).

O Brasil é o único dos três países que não explora o potencial pesqueiro da espécie. Conforme apresentado por Madureira et al (2009), a Argentina é a pioneira na explotação de anchoita e manufatura de diferentes tipos de produtos para consumo humano e - Uruguai apresenta uma produção inconstante ao longo dos anos, com períodos de fechamento das fábricas, sendo que o processamento da anchoita é 
voltado principalmente para produção de farinha e exportação para países como Alemanha, Itália, Rússia e China (Madureira et al., 2009).

Os resultados do Programa REVIZEE (Levantamento dos Recursos Vivos da Zona Econômica Exclusiva do Brasil), sintetizados em MMA (2006), mostram que a produção dos recursos pesqueiros brasileiros já explotados não deve aumentar e, para os estoques subexplotados, podese inferir um aumento bastante restrito na produção, sendo que, apenas a anchoita, apresenta potencial significativo de aproveitamento comercial.

No Brasil, a última estimativa da biomassa de anchoita indicam 675.500 toneladas, o que representa uma potencial de captura a partir de uma exploração sustentável do estoque de 135.000 toneladas anuais. Em 2005, um projeto financiado pelo Conselho Nacional de Desenvolvimento Científico e Tecnológico do Brasil (CNPq) confirmou a abundância e propiciou a geração de quatro protótipos de produtos baseados em anchoita: risoto desidratado, filé fermentado, sopa desidratada e salsicha. Isto demonstra o potencial para o desenvolvimento de produtos alternativos que podem gerar novos mercados. Os altos conteúdos protéicos da espécie tornam estes produtos adequados aos programas sociais do governo para combater a pobreza, como alimento para escolas e hospitais (Madureira et al., 2009).

$\mathrm{O}$ aumento da demanda por produtos a base de anchoita, causada pelo colapso de estoques de espécies co-genéricas em países como França, Espanha e Japão (FAO, 2007a,b), gerará uma busca por outros fornecedores para suprir o mercado, o que sugere a grande probabilidade de aumentar, nos próximos anos, a pressão pesqueira sobre o estoque de anchoita levando-o a ser explorado comercialmente no Brasil. Este fato também pode ser sustentado pela necessidade de suprir o desabastecimento de pescado causado pelo colapso de muitas pescarias demersais da região sudeste e sul (Haimovici et al., 2006) e a diminuição de estoques de outros pequenos pelágicos como a sardinha-verdadeira (Jablonski, 2007). O Brasil atualmente importa cerca de 60.000 toneladas de sardinha anualmente para suprir o mercado interno (Madureira et al., 2009).

A eminência de pescaria de anchoita no Brasil gera preocupação com o futuro manejo pesqueiro da espécie, e monitorar a abundância ou biomassa dos peixes é uma das prioridades em qualquer manejo. A bio-ecologia da anchoita, assim como de outros pequenos pelágicos, faz com que esta espécie seja particularmente sensível a forçantes ambientais (Agostini, 2000), o que gera consideráveis flutuações anuais em sua biomassa. Com isso, são necessárias avaliações mais freqüentes e contínuas, pois decisões de manejo precisam ser tomadas em curto prazo e podem envolver substanciais mudanças no padrão de explotação (King, 1995).

Dentre as técnicas disponíveis para avaliação de biomassa, o método hidroacústico é referido como o mais eficaz quando há necessidade de um conhecimento em tempo real a respeito de espécies pelágicas de curto ciclo de vida, ou para estoques subexplotados (Bertrand et al., 2004). No Brasil, os primeiros cruzeiros científicos para estimativas de abundância de anchoita aplicando-se a metodologia hidroacústica foram realizados em 1980 (Castello et al., 1991).

O elevado custo do "tempo de navio no mar" torna fundamental a otimização do tempo de cruzeiro científico. Um cruzeiro bem desenhado simplifica a análise dos dados e aumenta a chance de produzir resultados satisfatórios e, embora a tecnologia atual proveja equipamentos acústicos confiáveis, estes devem ser usados com entendimento dos fatores que determinam seu desempenho (Simmonds et al., 1992).

Este trabalho define e descreve o desenho estratégico do cruzeiro de estimativa de abundância mais adequado para a metodologia acústica, a ser aplicado à $E$. anchoita em águas brasileiras. Elementos como a estrutura do desenho do cruzeiro, época e freqüência de realização e aspectos relacionados aos equipamentos acústicos foram avaliados.

\section{PLANEJAMENTO DE UM CRUZEIRO}

De acordo com Cochran (1977), Simmonds et al. (1992) e Simmonds \& MacLennan (2005), o adequado planejamento de um cruzeiro deve seguir as seguintes etapas: 1) definição dos objetivos do cruzeiro; 2) determinação da unidade de estoque, ou seja, os limites de um estoque e, conseqüentemente, a área geográfica e o período amostral; 3) estimar o tempo de navio em relação à densidade amostral; 4) 
definir os dados a serem coletados e calcular o tempo disponível para o cruzeiro, levando em conta amostragens biológicas, dados hidrográficos ou de calibrações dos instrumentos; 5) decidir a estratégia de amostragem e o tipo de desenho amostral a ser seguido, necessariamente baseados no grau de precisão requerida; 6 ) definir os métodos de medição, que incluem a configuração da ecossonda e determinação dos equipamentos auxiliares; 7) expor a extensão calculada do cruzeiro em um mapa, assegurando que amostras representativas serão coletadas em toda a área e, 8) organizar a logística do trabalho de campo.

O tempo investido em um cruzeiro de pesquisa pode ser decidido baseando-se no entendimento de como a precisão dos resultados depende do espaçamento das transectas, que são as linhas percorridas pelo navio durante a coleta de dados. Aglen (1983) apresenta os resultados em termos de Coeficiente de Variação (CV) para expressar a variabilidade da média e sugere que $C V$ depende do Grau de Cobertura (GC) do cruzeiro, definido por $\mathrm{D} / \sqrt{ } \mathrm{A}$, onde $\mathrm{A}$ é a área sendo amostrada e $\mathrm{D}$ é a distância total de navegação do cruzeiro. Para planejamentos de cruzeiros acústicos, Simmonds \& MacLennan (2005) assumem que o grau de cobertura não deve ser inferior a 4 e quando este é maior que 6 , o erro amostral na estimativa de abundância tende a uma distribuição Gaussiana, o que simplifica o cálculo dos limites de confiança.

Nos desenhos prévios de cruzeiros deve se definir a forma como o conjunto de transectas será distribuído na área de estudo, que pode ter uma distribuição randômica (aleatória) ou sistemática, onde as transectas são distribuídas com um espaçamento fixo entre elas. A amostragem randômica traz simplicidade aos cálculos estatísticos e permite calcular estimadores imparciais da média e sua variância (Jolly \& Hampton, 1990), entretanto, pode deixar grandes porções da área sem amostrar, além de perfis muito próximos uns dos outros, o que é particularmente contraproducente para amostrar organismos com distribuição agregada (contagiosa). Se as pesquisas objetivam fornecer tendências interanuais de abundância, teremos estimativas mais corretas aplicando o mesmo espaçamento entre transectas a cada ano e, embora a abundância média de um ano contenha vícios, as diferenças entre anos serão imparciais se houver continuidade (ICES, 2004).

Dentro de uma distribuição de esforço sistemática, há duas possibilidades de desenho: os triangulares (ziguezague) e os retangulares (paralelos). Rivoirard et al. (2000) sustentam que desenhos paralelos são mais eficientes para transectas com comprimento maior que duas vezes o espaçamento, e triangulares, quando não for este $o$ caso.

\section{MATERIAIS E MÉTODOS}

Primeiramente realizou-se uma revisão nos estudos voltados ao planejamento de cruzeiros de pesquisa, conhecimento sobre a biologia da espéciealvo e aspectos ambientais da área de estudo que podem influenciar no comportamento, distribuição e abundância desta espécie.

Uma análise crítica foi realizada quanto a metodologia de obtenção de dados de todos os cruzeiros voltados à estimativas de abundância de anchoita na região sul do Brasil, que incluem quatro campanhas da série AREPE, entre 1980 e 1990 e três da série ECOPEL (Lima \& Castello, 1995), além de cruzeiros realizados em agosto e setembro de 2005, denominados A, descritos por Madureira et al. (2005). Para efeito de comparação, foi analisado o padrão de cruzeiros acústicos de estimativas anuais de biomassa de anchoita que vem sendo realizado na Zona Comum de Pesca Argentino - Uruguaia (ZCPAU), desde 1993, por equipes do Instituto Nacional de Investigação de Desenvolvimento Pesqueiro (INIDEP).

\section{RESULTADOS E DISCUSSÕES}

\section{Análise dos cruzeiros realizados na área de estudo}

As campanhas da série AREPE, realizadas entre o Cabo de Santa Marta e o Chuí, tiveram desenhos amostrais pré-planejados, compreendendo transectas paralelas à costa, de espaçamento variável, randomicamente selecionado e préestratificado, com maior esforço ao sul de Rio Grande. Os desenhos das campanhas da série ECOPEL basearam-se nos resultados do AREPE, em que a anchoita se distribuiu principalmente na área ao sul de Rio Grande ( $\left.32^{\circ} \mathrm{S}\right)$. Os cruzeiros não tiveram a continuidade necessária para um exaustivo 
acompanhamento da espécie na área, além de não cobrirem toda a área de distribuição, mas podem ser considerados estudos-piloto da distribuição e abundância da espécie na região sul do Brasil, fornecendo subsídios para a continuidade de avaliações anuais de biomassa.

Estimativas da abundância de anchoita na costa sul brasileira, ao sul de Rio Grande, voltaram a ser obtidas em 2005, com os cruzeiros ANCHOITA, gerando uma descontinuidade de 15 anos nas informações. O objetivo específico do cruzeiro não foi a estimativa de biomassa da espécie, mas estes apresentaram adequado grau de cobertura da área $\mathrm{e}$ os resultados destes cruzeiros comprovaram a abundância da espécie na região. Porém, a partir da análise do mapa de ocorrência de anchoita, pode-se perceber que os limites de distribuição desta espécie (Figura 1), tanto para o sul quanto para o norte, não foram atingidos, assim como o limite costeiro. Os limite sul não foi atingido por se estender sobre águas argentinas e uruguaias, já o limite norte foi estipulado devido a limitação de recursos para a realização do cruzeiro. O limite costeiro é um fator mais complexo de ser solucionado, devido a questões operacionais relacionadas à profundidade na região.

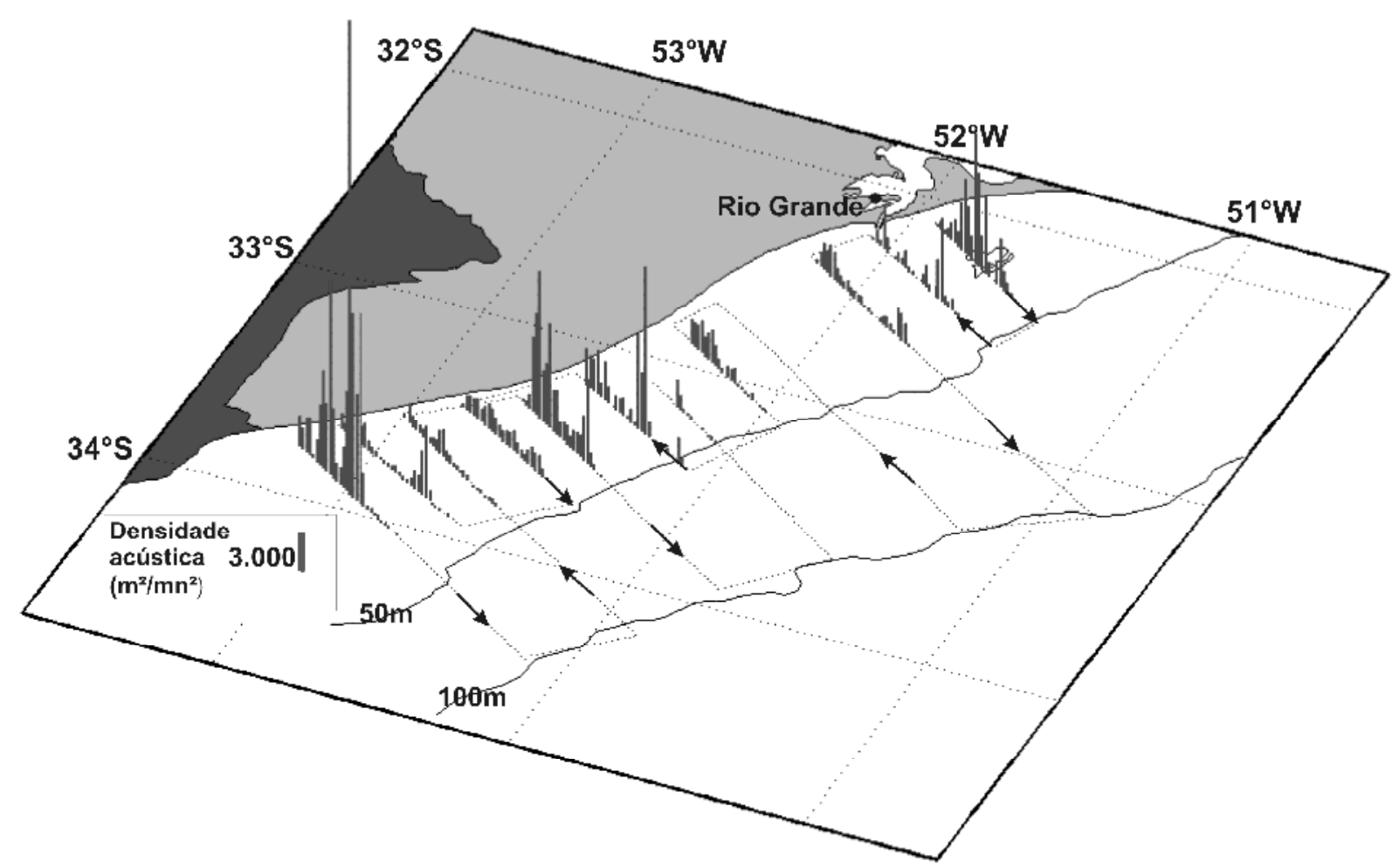

FIGURA 1 - Desenho do cruzeiro realizado no extremo sul do Rio Grande do Sul, em agosto de 2005 e distribuição da densidade acústica (NASC) de anchoita, representada pelas barras verticias (Madureira et al., 2005). As setas indicam o sentido da navegação.

$\mathrm{Na}$ costa uruguaia e argentina, entre $34^{\circ} \mathrm{e}$ $41^{\circ} \mathrm{S}$, os cruzeiros são realizados anualmente em outubro, considerada época de pico reprodutivo da espécie, sobre um desenho paralelo randômico préestratificado, com a divisão da área em seis estratos, feita de acordo com os dados históricos de distribuição da anchoita adulta (Hansen \& Madirolas, 1996). Este desenho estratificado de cruzeiro utilizado pelo
INIDEP é justificado devido ao maior conhecimento a respeito de características da distribuição da espécie, como diferenças em abundância e padrões de tamanho apresentados pela população. Estratificações tão minuciosas como ocorrem na região bonaerense ainda não são possíveis na costa sul-brasileira, pelo limitado número de cruzeiros e pelo fato de haver uma variação sazonal na abundância de anchoita, 
relacionada a fatores ambientais e hidrográficos que condicionam a migração de águas argentinas e uruguaias (Castello, 2005).

Todos os cruzeiros analisados foram realizados ininterruptamente durante períodos diurnos e noturnos, o que reduz o tempo de mar $e$ conseqüentemente, os custos com o cruzeiro. Porém, para espécies como a anchoita, que realizam migrações diárias, desfazendo os densos cardumes encontrados na coluna d'água durante o dia para dispersar-se próximo à superfície durante a noite (Soares et al., 2005), esta metodologia pode levar a erros de subamostragem da população devido a zona não amostrada na superfície. Esta zona, chamada de "Near Field", corresponde ao calado da embarcação somado a uma zona logo abaixo do transdutor da sonda e é formada pela a expansão e retração da onda acústica (Johannesson \& Mitson, 1983).

\section{Planejamento do cruzeiro acústico: o desenho amostral}

O objetivo do cruzeiro planejado é a estimativa anual de biomassa de Engraulis anchoita na costa sul do Brasil, a partir de metodologia hidroacústica, comparável entre anos, visando identificar correlações com outros fatores de influência na área, como distribuição de massas de água, além da análise da influência de uma futura pescaria na abundância do estoque.

A área amostral considerada estende-se desde - Chuí (3340'S) até o Cabo de Santa Marta Grande $\left(28^{\circ} \mathrm{S}\right)$. O limite costeiro do cruzeiro em projeção foi determinado para a isóbata de $15 \mathrm{~m}$ como uma tentativa de obter informações dos cardumes mais próximos à costa e o limite oceânico fixado foi de 100 $\mathrm{m}$, de acordo com a profundidade máxima de ocorrência de anchoita em outros cruzeiros realizados na região. Para determinação do limite costeiro foi levado em consideração questões de segurança para navegação e limite de profundidade de operação da rede de pesca considerada.

Simmonds et al. (1992) recomenda o desenho paralelo sistemático, com estratificações, caso se tenham distribuições não estacionárias, quando a densidade em uma parte da área é muito maior que em outra. Dessa forma a variância é reduzida e a precisão da estimativa aumentada, devido a maior homogeneidade dos dados dentro do estrato. Considera-se a distribuição da anchoita como contagiosa, pois a partir dos mapas de distribuição de cruzeiros são percebidas regiões de alta abundância, circundadas por regiões de abundância praticamente nula, justificando o uso de um desenho sistemático. Resultados de cruzeiros anteriores mostram que a densidade ao sul de Rio Grande é maior que ao norte, levando a consideração de uma estratificação do cruzeiro em relação a estas duas áreas, exercendo maior esforço amostral no sul, onde pode haver maior abundância. A partir da análise da largura da plataforma e a relação entre comprimento das transectas e espaçamento entre estas, recomenda-se um desenho com transectas paralelas entre si.

Além da distribuição anisotrópica em relação às isobatas, que levam a aplicação de um desenho com transectas perpendiculares à costa, há evidências de mudanças temporais na distribuição dos cardumes, devido ao ciclo migratório anual da anchoita. Durante o inverno, uma fração do estoque migra de águas argentinas e uruguaias para o Brasil, onde há condições favoráveis de desova e alimentação, voltando para águas costeiras e intermediárias argentinas no fim da primavera, pico da estação reprodutiva (Lima \& Castello, 1995). Considera-se a estação de pico reprodutivo da espécie na região sul brasileira, correspondente aos meses de inverno, como a época mais adequada a levantamentos acústicos de abundância. O melhor período seria o de "janela de estabilidade" do estoque, no entanto, o período exato em que a anchoita entra na região e que começa a migração de retorno para o sul não é conhecido. A estratégia sugerida a fim de diminuir erros com duplicidade na amostragem de cardumes é direcionar o trabalho no sentido contrário a migração do estoque durante esta época do ano, ou seja, de norte para sul.

Devido à dispersão noturna da anchoita, as estimativas acústicas devem ser feitas somente durante o dia, dedicando o período noturno para outras atividades, como amostragem de plâncton e execução de estações hidrográficas.

A área de cada um dos dois estratos, norte e sul de Rio Grande, foi calculada a partir de ferramentas de medição de área do software ArcGis 9.2 e, a partir da técnica de Aglen (1983), com grau 
de cobertura seis, estimou-se o comprimento do cruzeiro. O número de transectas foi encontrado dividindo o comprimento do cruzeiro pelo comprimento médio destas, estimado a partir do cálculo da média das medições feitas entre as isóbatas de 15 e $100 \mathrm{~m}$ sobre uma carta náutica. O espaçamento entre transectas encontrado foi menor na área sul.

Para diminuir os vícios relacionados às estimativas da abundância inter-anuais, sugere-se inserir um elemento de randomização em relação ao ponto inicial da grade amostral, fazendo com que a distância entre a transecta e a borda da área de cada um dos estratos seja uma proporção randômica (0$100 \%$ ) do espaçamento.

A tabela 1 apresenta os fatores considerados para o desenho do cruzeiro e a figura 2, o desenho do cruzeiro plotado em uma carta náutica. $O$ porto de Rio Grande foi considerado base de partida e chegada dos cruzeiros.

TABELA 1 - Parâmetros do desenho de cruzeiro sugerido para estimativa anual de biomassa de anchoita na região sul do Brasil, utilizando metodologia acústica.

\begin{tabular}{ccc}
\hline & Estrato 1 - Norte & Estrato 2 - Sul \\
\hline Limites latitudinais & $28^{\circ} 35^{\prime}-32^{\circ} 00^{\prime}$ S & $32^{\circ} 00^{\prime}-33^{\circ} 45^{\prime}$ S \\
Limites batiméticos $(\mathrm{m})$ & $15-100$ & $15-100$ \\
Área total $\left(\mathrm{mn}^{2}\right)$ & 18.100 & 12.559 \\
Grau de cobertura & 6 & 6 \\
Comprimento total da ecointegração $(\mathrm{mn})$ & 810 & 673 \\
Comprimento médio das transectas $(\mathrm{mn})$ & 42 & 65 \\
Número de transectas & 19 & 10 \\
Espaçamento entre transectas $(\mathrm{mn})$ & 14 & - \\
Distância de navegação porto - início cruzeiro $(\mathrm{mn})$ & 280 & 120 \\
Distância de navegação fim cruzeiro $(\mathrm{mn})-$ porto & - & 110 \\
\hline
\end{tabular}




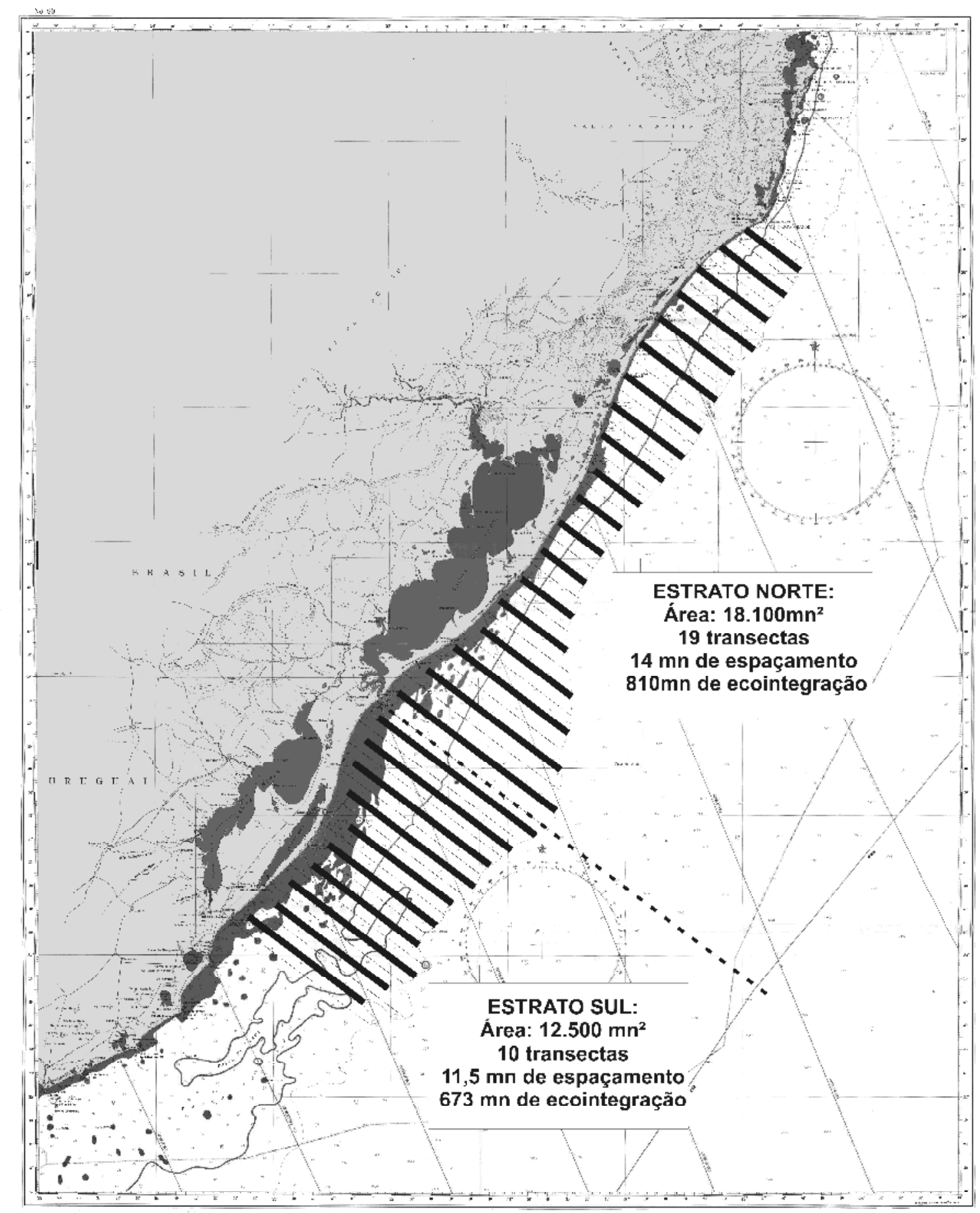

FIGURA 2 - Desenho de cruzeiro sugerido para estimativas de biomassa de anchoita no sul do Brasil, utilizando metodologia acústica, plotado em uma Carta N90 digital, da"llha de Santa Catarina a Mandonado", disponibilizada pela Marinha do Brasil. A zona sombreada indica regiões marinhas e outros corpos d'água rasos (profundidade local menor ou igual a 20 metros).

Além do tempo de prospecção acústica, outras atividades devem compor os cálculos do tempo total do cruzeiro, como: embarque e desembarque, deslocamento entre o porto e a área de estudo, amostragens de plâncton, pesca para identificar ecotraços e estações hidrográficas. A tabela 2 apresenta o tempo necessário para a realização de cada uma das atividades, considerando 10 nós a velocidade do navio. As intertransectas devem ser desconsideradas da sequência de pesquisa e sempre que possível, devem ser realizadas durante a noite. 
TABELA 2 - Cômputo do tempo necessário para cada atividade no cruzeiro de estimativa anual de biomassa de anchoita na região sul do Brasil, utilizando metodologia acústica (D: diurno; N: noturno).

\begin{tabular}{|c|c|c|c|c|}
\hline \multirow{2}{*}{ Atividade } & \multicolumn{2}{|c|}{ Estrato Norte } & \multicolumn{2}{|c|}{ Estrato sul } \\
\hline & Distância & Período & Distância & Período \\
\hline Embarque & 1 dia & $\mathrm{D}$ & 1 dia & $\mathrm{D}$ \\
\hline Navegação Rio Grande - Área amostral & $280 \mathrm{mn}$ (28 horas) & $\mathrm{N} / \mathrm{D}$ & \multicolumn{2}{|r|}{+} \\
\hline Navegação Área amostral - Rio Grande & \multicolumn{2}{|l|}{-} & $120 \mathrm{mn}$ (12 horas) & $\mathrm{N}$ \\
\hline Prospecção Acústica (transectas) & $\begin{array}{c}19 \text { transectas } \\
810 \mathrm{mn} \text { (81horas) }\end{array}$ & D & $\begin{array}{c}10 \text { transectas } \\
673 \mathrm{mn} \text { (68 horas) }\end{array}$ & D \\
\hline Intertransectas & $\begin{array}{l}19 \text { intertransectas } \\
\text { (270mn - } 27 \text { horas) }\end{array}$ & $\mathrm{N}$ & $\begin{array}{c}10 \text { intertransectas } \\
(115 \mathrm{mn}-11,5+\text { horas })\end{array}$ & $\mathrm{N}$ \\
\hline Estações de CTD & 57 (28,5 horas) & $1 / 3(\mathrm{D}), 2 / 3(\mathrm{~N})$ & 30 (15 horas) & $1 / 3(\mathrm{D}), 2 / 3(\mathrm{~N})$ \\
\hline Pesca & 38 (57 horas) & $\mathrm{D}$ & 20 (30 horas) & $\mathrm{D}$ \\
\hline Horas de trabalho diurno & 175 & & 114 & \\
\hline Horas de trabalho noturno & 65,5 & & 33,5 & \\
\hline Média de transectas/dia & $1,2 / \mathrm{dia}$ & & 1/dia & \\
\hline Tempo total do cruzeiro & 16 dias & & 10 dias & \\
\hline Tempo de salvaguarda & 3 dias & & 2 dias & \\
\hline Tempo total por etapas & 20 dias & & 13 dias & \\
\hline TEMPO TOTAL & & & & \\
\hline
\end{tabular}

Habiaga et al. (1997) indicam a rede de meiaágua como o petrecho que melhor corresponde às necessidades de amostragem em toda a coluna d'água. Para fins de planejamento, foram fixados dois arrastos por transecta, de 30 minutos cada, que somado ao tempo médio de largada e recolhimento da rede e de retorno ao local de recomeço da integração, totalizaram 1 hora e 30 minutos. As estações hidrográficas utilizando um sensor de condutividade, temperatura e profundidade, CTD (Conductivity, Temperature and Depth Sensor), devem ser feitas no início, meio e fim das transectas e considera-se necessário 30 minutos para cada. Um tempo extra de precaução, no caso de mal tempo e eventuais reparos durante o cruzeiro, foi inserido no cálculo, correspondendo a $20 \%$ do tempo total.

\section{Planejamento do cruzeiro: os parâmetros acústicos}

Uma ecossonda científica é o equipamento básico para aquisição de dados acústicos e estimativa de biomassa (Simmonds \& MacLennan, 2005; Madureira \& Rossi-Wontschowski, 2005). Ela pode operar com várias freqüências, fato que melhora a acurácia na identificação dos registros das espécies. Atualmente a ecossonda científica utilizada tanto no navio oceanográfico da FURG, quanto do INIDEP é da marca SIMRAD, modelo EK 500, no entanto, já existem disponíveis no mercado modelos mais avançados. Uma sonda de rede também é um equipamento essencial para cruzeiros acústicos, com o objetivo de monitorar a abertura da rede e a eficiência desta na pesca.

Além destes equipamentos essenciais, é importante a presença de sonar para acompanhamento de reações horizontais de afungentamento da espécie devido a presença do navio e de um Perfilador Doppler Acústico de Corrente, ADCP (Acoustic Doppler Current Profiler), para a análise de velocidade e direção de correntes.

A escolha da freqüência de operação envolve compromissos entre a necessidade de longo alcance e resolução entre alvos. Levando-se em consideração o comportamento de formação de extensos cardumes de anchoita propõe-se a utilização de $38 \mathrm{kHz}$ para a obtenção de estimativas de biomassa dessa espécie, concomitantemente à freqüência de $120 \mathrm{kHz}$, para auxiliar no processo de categorização dos registros (Madureira et al., 1993). 
No caso de freqüências de $38 \mathrm{kHz}$, três configurações de duração de pulso são possíveis, o pulso curto (0,3 ms), médio ( $1 \mathrm{~ms})$ e longo ( $3 \mathrm{~ms})$. A duração de pulso influencia na resolução entre alvos, pois esta é igual à metade do comprimento de pulso. Pulsos mais curtos geram maior resolução entre alvos, porém, segundo Soule et al. (1997), valores do índice de reflexão individual, Target Strength (TS), obtidos com o pulso curto podem levar a resultados duvidosos em campo, pois dependem de fatores complexos, influenciados pelo limiar de TS. Para este cruzeiro, considera-se a duração de pulso de $1 \mathrm{~ms}$ como mais adequada.

As estimativas de biomassa pelo método de eco-integração (Madureira \& Rossi-Wontschowski, 2005) são dependentes equações de TS de cada espécie. No entanto, não existem estudos definitivos sobre o TS de Engraulis anchoita. Trabalhos que apresentaram resultados de estimativa de abundância de anchoita utilizaram equações de TS para espécies co-genéricas, mas diferentes equações resultarão em diferentes valores de biomassa. Há a necessidade de um maior número de pesquisas voltadas à obtenção de valores definitivos de TS para a anchoita, para que as estimativas de biomassa sejam mais acuradas. Estes valores poderiam ser obtidos nos cruzeiros durante a noite, quando os indivíduos encontram-se dispersos, sendo mais fácil a detecção de alvos individuais.

\section{CONCLUSÕES}

A anchoita é o único estoque de abundância determinada ainda virgem no Brasil. Esta condição encontra-se em modificação, com a comprovação da viabilidade da pesca e geração de produtos que podem suprir o mercado interno e para exportação. Os colapsos de outros estoques de pequenos pelágicos fazem com que aumente a preocupação por um adequado manejo da espécie, o que resultaria em um dos únicos estoques manejados desde o inicio da explotação. Devido a variabilidade interanual do estoque, um adequado manejo requer levantamentos contínuos da abundância do recurso.

A revisão dos cruzeiros de estimativa de biomassa de anchoita na região sul brasileira demonstra a ausência de pesquisas continuadas e a necessidade do planejamento de cruzeiros visando o acompanhamento de modificações na abundância do estoque. As informações disponibilizadas pelos cruzeiros permitem o planejamento de um cruzeiro de estimativa de biomassa para a espécie, mas demonstram a falta de determinadas informações a respeito do estoque necessárias para subsidiar aumento da precisão dos trabalhos, tais como comportamento de migração horizontal, e determinação de um TS próprio para a espécie.

O desenho de cruzeiro apresentado representa uma proposta imediata de avaliação da biomassa do estoque encontrado em águas brasileiras. $\mathrm{Na}$ Argentina e Uruguai, como resultado do interesse pesqueiro na espécie, as estimativas de abundância de anchoita vêm sendo obtidas por cruzeiros acústicos desde 1993 e 1994, realizadas por equipes do INIDEP (Instituto Nacional de Investigação de Desenvolvimento Pesqueiro, Argentina), com um caráter participativo com o Uruguai (DINARA, Direção Nacional dos Recursos Aquáticos), para o estoque adulto e recrutante, respectivamente. $O$ fato da metodologia aplicada por estes países ser equivalente a proposta aqui, facilita a implementação do monitoramento de todo o estoque bonaerense. Um adequado conhecimento do estoque só será possível a partir de trabalhos conjuntos dos três países de forma a planejar cruzeiros que cubram toda a área de distribuição da espécie.

\section{REFERÊNCIAS}

AGLEN, A. 1983. Random errors of acoustic fish abundance estimates in relation to the survey grid density applied FAO Fisheries Report, 300: 293-298.

AGOSTINI, V. 2000. Relationships between small pelagics and the environment: Where have we been and where do we go from here? In: GFCM, 2000. Report of the 2000 Session of the Working Group on small pelagic species. Scientific Advisory Committee-GFCM. Fuengirola, Spain 1 a 3 de Março de 2000.

BAKUN, A \& RH PARRISH. 1991. Comparative studies of coastal pelagic fish reproductive habitats: the anchovy (Engraulis anchoita) of southwestern Atlantic. ICES Journal of Marine Science. 48: 343-361.

BERTRAND, A, M SEGURA, M GUTIÉRREZ \& L VÁSQUEZ. 2004. From small-scale habitat loopholes to decadal cycles: a habitatbased hypothesis explaining fluctuation in pelagic fish populations off Peru. Fish and Fisheries, 5: 296-316.

CASTELLO, JP. 2005. A anchoita Engraulis anchoita (Engraulididae, Teleosteii) no sul do Brasil. Análise/Refinamento dos Dados Pretéritos Sobre Prospecção Pesqueira. In: HAIMOVICI, M. 
(coord.) 2005. Avaliação do Potencial Sustentável de Recursos Vivos na Zona Econômica Exclusiva - REVIZEE. 31 (3) Anex. 2. $61 \mathrm{p}$.

CASTELLO, L \& JP CASTELLO. 2003. Anchovy stocks (Engraulis anchoita) and larval growth in SW Atlantic. Fisheries Research, 59: 409-421.

CASTELLO, JP, RP HABIAGA, JC AMARAL \& ID LIMA JR. 1991. Prospecção hidroacústica e avaliação da biomassa de sardinha e anchoita, na região sudeste do Brasil (out./nov. de 1988). Publicação do Instituto Oceanográfico, SP, 8: 15-29.

COCHRAN, WG. 1977. Sampling techniques. 3 ed. New York: John Willey.

FAO. 2007a. FISHSTAT Plus. Universal software for fishery statistical time series. Rome, Italy.

FAO. 2007b. The State of World Fisheries and Aquaculture 2006, Rome, Italy, 162p.

HABIAGA, R, C SOARES, L MADUREIRA \& D ELISEIRE JR. 1997. Rede de meia água: Operação e eficiência como amostrador em cruzeiros de ecointegração. Atlântica, 19: 145-159.

HAIMOVICI, M, JP CASTELLO \& CM VOOREN. 1997. Fisheries. In: SEELIGER, U, C ODEBRECHT \& JP CASTELLO. Subtropical Convergence Environments. The coast and sea in the Southwestern Atlantic. Springer, 183-196.

HAIMOVICI, M, M VASCONCELLOS, D KALIKOSKI, P ABDALAH, JP CASTELLO \& D HELLEMBRANDT. 2006. Diagnóstico da pesca no litoral do estado do Rio Grande do Sul. In: ISAAC, VN, M HAIMOVICI, SA MARTINS \& JM ANDRIGUETTO (Org). 2006. A pesca marinha e estuarina do Brasil no início do século XXI: recursos, tecnologias, aspectos socioeconômicos e institucionais. Belém; UFPA, 157-180.

HANSEN, JE \& A MADIROLAS. 1996. Distribuicion, evaluacion acustica y estructura poblacional de la anchoita. Resultados de las campañas del año 1993. Revista de Investigacion y Desarrollo Pesquero, 10. 5-21.

ICES. 2004. Report of the Workshop on Survey Design and Data Analysis (WKSAD). 21-25 June 2004. Aberdeen, UK. ICES Fisheries Technology Committee. ICES. Council Meeting Papers, 07: $261 \mathrm{p}$.

JABLONSKI, S. 2007. The Brazilian sardine. Is there any room for modelling? Pan-American Journal of Aquatic Sciences, 2 (2): 86-93.

JOHANNESSON, KA, \& RB MITSON. 1983. Fisheries acoustics: a practical manual for aquatic biomass estimation. FAO Fisheries Technical Paper, 240: $249 \mathrm{p}$.

JOLLY, G.M. \& I HAMPTON. 1990. A stratified random transect design for acoustic surveys of fish stocks. Canadian Journal of Fisheries and Aquatic Sciences, 47: 1282-1291.

KING, M. 1995. Fisheries biology: assessment and management. Fishing New Books. 341 p.

LIMA, ID \& JP CASTELLO. 1995. Distribution and abundance of South-west Atlantic anchovy spawners (Engraulis anchoita) in relation to oceanographic process in the southern Brazilian shelf. Fisheries Oceanography. 4(1):1-16.
MADUREIRA, LSP, P WARD \& A ATKINSON. 1993. Differences in backscattering strength determined at 120 and $38 \mathrm{kHz}$ for three species of Antartic macroplankton. Marine Ecology Progress Series. 9: 17-24.

MADUREIRA, LASP, JP CASTELLO, MI QUEIROZ, C PRENTICEHERNÁNDEZ, WA RUIZ, MLPE SANTO, PR ABDALLAH, PR SCHWINGEL. 2005. Projeto: Pesca de anchoita (Engraulis anchoita) com rede de meia água na plataforma continental da região sudeste/sul do Brasil, processamento do pescado em terra e análise econômica da viabilidade da atividade CNPq/ SEAP-PR/FURG. Relatório de atividades CT-Agro 504.544/2003-9. 122 p.

MADUREIRA, LSP \& CLD ROSSI-WONTSCHOWSKI (eds.). 2005. Prospecção de recursos pesqueiros pelágicos na Zona Econômica Exclusiva da Região Sudeste-Sul do Brasil: hidroacústica e biomassa. São Paulo: Instituto Oceanográfico USP, Série documentos REVIZEE: Score Sul, 144p.

MADUREIRA, LASP, JP CASTELLO, C PRENTICE-HERNÁNDEZ, MI QUEIROZ, ML SANTO, WA RUIZ, P ABDALLAH, J HANSEN, MI BERTOLOTTI, E MANCA S, MI YEANNES, N AVDALOV \& S AMORIN. 2009. Current and potential alternate food use of the Argentine anchoita (Engraulis anchoita) in Argentina, Uruguay and Brazil. In M.R. Hasan (ed). Fish as feed inputs for aquaculture: practice, sustainability and implications. FAO Fisheries and Aquaculture Technical Paper. No. 518. Rome, FAO.

MMA. 2006. Programa REVIZEE: avaliação do potencial sustentável de recursos vivos na zona econômica exclusiva: relatório executivo. Ministério do Meio Ambiente. Secretaria de Qualidade Ambiental. Brasília, 280p.

RIVOIRARD, J, J SIMMONDS, KG FOOTE, PG FERNANDES \& N BEZ. 2000. Geostatistics for Estimating Fish Abundance, Blackwell Science, Oxford, 206p.

SIMMONDS, EJ \& DN MACLENNAN. 2005. Fisheries acoustics. Chapman \& Hall. Fish and Fisheries Series.

SIMMONDS, EJ, NJ WILLIAMSON, F GERLOTTO, \& A AGLEN. 1992. Hydroacoustic survey design and analysis procedure: a comprehensive review of current practice. ICES Cooperative Research Report, 187: $112 \mathrm{p}$.

SOARES, CF, LSP MADUREIRA, RP HABIAGA, LD LAURINO, CS FERREIRA \& SC WEIGERT. 2005. Caracterização dos ecotipos detectados durante os cruzeiros de prospecção pesqueira acústica entre Cabo de São Tomé (RJ) e Chuí (RS). In: MADUREIRA, LSP, CLD ROSSI-WONTSCHOWSKI (eds.). 2005. Prospecção de recursos pesqueiros pelágicos na Zona Econômica Exclusiva da Região Sudeste-Sul do Brasil: hidroacústica e biomassa. São Paulo: Instituto Oceanográfico USP, Série documentos REVIZEE: Score Sul. 144p.

SOULE, M., M BARANGE, H SOLLI \& I. HAMPTON. 1997. Performance of a new phase algorithm for discriminating between single and overlapping echoes in a split-beam echo sounder, ICES Journal of Marine Science, 54: 934-938. 